\title{
The Plasma LncRNA Acting as Fingerprint in Hilar Cholangiocarcinoma
}

\author{
Jian Shia,b Xiaohua Lic Fan Zhang ${ }^{\mathrm{a}} \quad$ Linqun Kong $^{\mathrm{a}} \quad$ Xingyuan Zhang ${ }^{\mathrm{a}}$ \\ Yu Cheng ${ }^{a}$ Qinghai Guan ${ }^{a}$ Xuefeng Cao ${ }^{a}$ Wentao Zhu ${ }^{a}$ Kun Ou \\ Qiangpu Chen ${ }^{\mathrm{a}}$ Sanyuan $\mathrm{Hu}^{\mathrm{b}}$ \\ aDepartment of Hepatobiliary Surgery, Binzhou Medical University Hospital, Binzhou, bepartment \\ of General Surgery, Qilu Hospital of Shandong Universiy, Jinan, 'Department of Pediatrics, Binzhou \\ Medical University Hospital, Binzhou, China
}

\section{Key Words \\ Cancer $\bullet$ Sensitivity $\bullet$ Specificity $\bullet$ Risk score $\bullet$ Circulating}

\begin{abstract}
Background \& Aims: Current studies have indicated that long non-coding RNAs (IncRNAs) could act as tumor biomarkers for disease diagnosis and prognosis prediction. In this study, we mainly focused on determining the expression of circulating IncRNAs in patients suffering for hilar cholangiocarcinoma $(H C)$, aiming to reveal the potential IncRNA as a fingerprint. Methods: A total 12 IncRNAs were previously proven to be aberrantly expressed in HC tumor tissues. All of the 12 IncRNAs were selected as candidate targets for subsequent circulating IncRNA assay. The candidate IncRNAs were validated by qRT-PCR arranged in training and validation sets. The risk score analysis was employed. Data was presented with receiver operating characteristic curve (ROC). Results: Circulating PCAT1, MALAT1, and CPS1-IT1 were significantly increased in plasma samples of $\mathrm{HC}$ patients in both the training set and validation set. Through ROC analysis, we found that the three plasmatic IncRNAs presented the area under ROC curve value (AUC) as $0.784,0.860$, and 0.677 . Further combination with the three factors indicated a higher power (AUC, 0.893; sensitivity, 85.5\%; specificity, 93.2\%). Conclusion: This was the first time to reveal the potential circulating fingerprints for predicting HC. PCAT1, MALAT1, and CPS1-IT1 may act as novel early diagnosis biomarkers for predicting HC.
\end{abstract}

\section{Introduction}

(C) 2018 The Author(s)

Published by S. Karger AG, Basel

Cholangiocarcinoma accounts for approximately $10 \%$ of primary hepatic tumors and is divided into intrahepatic cholangiocarcinoma (ICC) and extrahepatic cholangiocarcinoma (ECC) $[1,2]$. Nearly $80 \%-90 \%$ of cholangiocarcinomas are of extrahepatic origin and are divided into perihilar (Klatskin tumors) and distal tumors based on their location [3,4].

J. Shi and X. Li contributed equally to this work. 
Another subgroup is Hilar cholangiocarcinoma (HC). Despite recent improvements in cholangiocarcinoma treatment, surgical resection and transplantation is still the main therapy, as this can cure early-staged patients $[5,6]$. Unfortunately, there are no specific biomarkers or unique clinical manifestations for cholangiocarcinoma, meanwhile, HC is typically characterized by advanced stage diagnosis. Therefore, novel diagnostic or therapeutic targets that underlie HC initiation and progression should be explored to better diagnose and cure HC.

HC patients have prognoses and limited therapeutic regimens [7, 8]. Thus, improving the diagnostic efficacy of $\mathrm{HC}$ is urgent and necessary, especially regarding biomarkers. Classic humoral cholangiocarcinoma markers such as carbohydrate antigen (CA)-199 and CA-125 are now regarded as insensitive and unspecific $[9,10]$. As gene expression patterns are greatly altered in $\mathrm{HC}$, it is urgent to identify new epigenetic biomarkers for HC patients.

With the progression of whole-genome sequencing technology, it has been found that $<2 \%$ of the transcribed mammalian genome is protein-encoding, while the rest is non-coding RNA [11-13]. Long non-coding RNAs (lncRNAs) are one type of non-protein encoding transcript, and are $>200 \mathrm{bp}$ in length $[14,15]$. Recently, many lncRNAs have been reported to play important roles in tumorigenesis, cancer progression and drug resistance [16-18]. Furthermore, evidence of circulating lncRNAs as biomarkers is rapidly increasing and has been applied to human diseases $[19,20]$. Researchers have recently identified that circulating lncRNAs might act as biomarkers for the early diagnosis of multiple human cancers, including hepatocellular carcinoma, gastric cancer, and prostate cancer [21, 22]. Most previous studies have focused on the promoting or inhibiting roles miRNAs might have on tumorigenic processes of malignancies such as HC [23]. However, more and more researchers have seen the potential of miRNAs as biomarkers for diagnosing, predicting, and monitoring the prognosis of HC patients. However, the potential predictive ability of IncRNAs in $\mathrm{HC}$ has not been fully investigated.

In this study, we identified lncRNAs that were differentially expressed in the tumor tissues of HC patients. In total, 12 IncRNAs (CCAT2, Sox2ot, UCA1, TUG1, AFAP1-AS1, CCAT1, NEAT1, PCAT1, PANDAR, MALAT1, H19, and CPS1-IT1) that were previously reported to have altered expression in $\mathrm{HC}$ were enrolled as candidate diagnostic makers. We hypothesized that these candidate lncRNAs might be released into circulation during HC initiation or progress and could be used to diagnose and monitor HC. Additionally, we aimed to determine lncRNAs that acted as fingerprints for the early identification of HC.

\section{Materials and Methods}

\section{Samples and screening phase}

All plasma samples were obtained from patients diagnosed with HC between July 2012 and June 2017 at Qilu Hospital of Shandong University and Binzhou Medical University Hospital. Control samples were from healthy volunteers without any health problems during their health check-ups at the aforementioned hospitals. This research protocol was approved by the Shandong University and Binzhou Medical University ethical review boards. Written informed consent was obtained from every participant. Peripheral blood samples of patients were collected before surgery. Blood samples were collected in a vacuum cube, followed by centrifugation at 5, $000 \mathrm{rpm}$ for $15 \mathrm{~min}$. All samples were stored at $-80^{\circ} \mathrm{C}$ until analysis. The relevant clinical data of all patients were available. All patients were diagnosed by histological examination. Detailed clinical information of patients and healthy controls are summarized in Table1.

The screening phase was divided into a training set and a validation set. Paired samples, including 20 $\mathrm{HC}$ patients and 20 healthy controls, were enrolled while the remaining 120 patients and 120 controls were used as the validation cohort. 


\section{Cellular Physiology Cell Physiol Biochem 2018;49:1694-1702 \begin{tabular}{l|l|l} 
and Biochemistry & Dublished online: 20 September, 2018 & $\begin{array}{l}\text { C } 2018 \text { The Author(s). Published by S. Karger AG, Basel } \\
\text { www.karger.com/cpb }\end{array}$
\end{tabular}}

\section{Training set}

All IncRNA candidates were tested in an independent cohort of 20 plasma samples obtained from patients. All patients were pathologically diagnosed with $\mathrm{HC}$ at the Qilu Hospital of Shandong University and Binzhou Medical University Hospital. Expression levels of these candidates were analyzed in all samples, and the comparative $2^{-\Delta \Delta \mathrm{Ct}}$ method was used for data presentation, and Student's t-test was used to analyze differences between patients and healthy controls.

\section{Validation set}

A case-control study was designed to validate differences in the relative expression levels of selected biomarker candidates in $120 \mathrm{HC}$ patients and 120 healthy controls (the validation cohort).

\section{Quantitative real-time PCR ( $q R T$-PCR)}

Three to five tubes of blood, each tube containing approximately $10 \mathrm{~mL}$ of blood, were used for plasma isolation. All tubes were EDTA-anticoagulation tubes. After centrifugation, we obtained approximately 20-30 $\mathrm{mL}$ of plasma for RNA extraction. Total RNA containing small RNAs was extracted from plasma using TRIzol reagent (Invitrogen, Carlsbad, CA, USA) and purified with the mirVana miRNA Isolation Kit (Ambion, Austin, TX, USA) according to the manufacturer's protocol. The purity and concentration of RNA samples were determined from 0D260/280 readings using a spectrophotometer (NanoDrop ND-1000, Thermo Fisher Scientific, Waltham, MA, USA). RNA integrity was determined by $1 \%$ formaldehyde denaturing gel electrophoresis. For mRNA detection, total RNAs (500 ng) were reverse transcribed using the reverse transcription kit and the Oligo-dT approach (with poly-A) or random hexamer primer method (without poly-A) (Takara, Tokyo, Japan). The primers used for RT-PCR are presented in Table 2. The SyberFreen technique was used for qRT-PCR, and was performed by using an ABI Prism 7900HT (Applied Biosystems, Foster City, CA, USA).

\section{Risk score analysis}

Risk score analysis was performed to evaluate associations between plasma lncRNA expression levels. The upper $95 \%$ reference interval of each IncRNA value in controls or the non-metastasis group was set as the threshold to code the expression level of the corresponding IncRNA for each sample as 0 and 1 in the training set. A risk score function (RSF) to predict $\mathrm{HC}$ was defined according to a linear combination of the expression level for each IncRNA. For example, the RSF for sample i using information from three lncRNAs was:
Table 1. The clinicopathological information in HC patients and healthy controls. a: Chi-square test

\begin{tabular}{|c|c|c|c|}
\hline Characteristics & $\mathrm{HC}$ & Control & P value ${ }^{a}$ \\
\hline All cases & 120 & 120 & \\
\hline \multicolumn{4}{|l|}{ Age } \\
\hline$<60$ & 85 & 88 & 0.666 \\
\hline$\geq 60$ & 35 & 32 & \\
\hline \multicolumn{4}{|l|}{ Gender } \\
\hline Male & 91 & 90 & 0.910 \\
\hline Female & 29 & 30 & \\
\hline \multicolumn{4}{|c|}{ Differentiation grade } \\
\hline Well & 33 & & \\
\hline Moderate & 61 & & \\
\hline Poorly & 26 & & \\
\hline \multicolumn{4}{|l|}{ Tumor Size $(\mathrm{cm})$} \\
\hline$\leq 5 \mathrm{~cm}$ & 89 & & \\
\hline$>5 \mathrm{~cm}$ & 31 & & \\
\hline \multicolumn{4}{|c|}{ Tumor Origination } \\
\hline Left & 66 & & \\
\hline Right & 49 & & \\
\hline Bilateral & 5 & & \\
\hline \multicolumn{4}{|l|}{ TNM stage } \\
\hline I-II & 41 & & \\
\hline III & 79 & & \\
\hline \multicolumn{4}{|l|}{ Metastasis } \\
\hline Yes & 98 & & \\
\hline No & 22 & & \\
\hline
\end{tabular}

Table 2. Primers for Quantitative real-time PCR

\begin{tabular}{lcc}
\hline Gene name & & Sequence \\
\hline CCAT2 & Forward Primer & AGACAGTGCCAGCCACC \\
& Reverse Primer & TGCCAAACCTTCCCTTA \\
Sox2ot & Forward Primer & GCTCGTGGCTTAGGAGATTG \\
& Reverse Primer & CTGGCAAAGCATGAGGAACT \\
UCA1 & Forward Primer & TTTGCCAGCCTCAGCTTAAT \\
& Reverse Primer & TTGTCCCCATTTCCATCAT \\
TUG1 & Forward Primer & TAGCAGTTCCCAATCCTTG \\
& Reverse Primer & CACAAATCCCATCATTCCC \\
AFAP1-AS1 & Forward Primer & ATGGGGTAACTCAAAAAGCCTG \\
& Reverse Primer & GCAGCAATTCAGAGCCAGTC \\
CCAT1 & Forward Primer & TCACTGACAACATCGACTTTGAAG \\
& Reverse Primer & GGAGAAAACGCTTAGCCATACAG \\
NEAT1 & Forward Primer & CCTGTCCGGCACCTCATAG \\
& Reverse Primer & GGCCTTTAGGCAGGCTCTT \\
PCAT1 & Forward Primer & AATGG CATGAACCTGGGAGGCG \\
& Reverse Primer & GGCTTTGGGAATGCTTTGGAG \\
PANDAR & Forward Primer & CTGTTAAGGTGGTGGCATTG \\
& Reverse Primer & GGAGGCTCATACTGGCTGAT \\
MALAT1 & Forward Primer & AGCGGAAGAACGAATGTAAC \\
& Reverse Primer & GAACAGAAGGAAGAGCCAAG \\
H19 & Forward Primer & ATCGGTGCCTCAGCGTTCGG \\
& Reverse Primer & CTGTCCTCGCCGTCACACCG \\
CPS1-IT1 & Forward Primer & CACAGATGATCCACGGCG TT \\
& Reverse Primer & GCGTGCATCAATGACACTTCA \\
GAPDH & Forward Primer & GGAGCGAGATCCCTCCAAAAT \\
& Reverse Primer & GGCTGTTGTCATACTTCTCATGG \\
& &
\end{tabular}


rsfi $=\sum 3 j-1 W j . s i j$. In the above equation, sij is the risk score for lncRNA $j$ on sample $i$, and $W j$ is the weight of the risk score of IncRNA $j$. The risk score of three lncRNAs was calculated using the weight by the regression coefficient that was derived from the univariate logistic regression analysis of each IncRNA. Samples were ranked according to their RSF and then divided into a high risk group, representing HC patients, and a low risk group, representing control individuals or non-metastatic patients. Frequency tables and receiver operating characteristic (ROC) curves were then used to evaluate the diagnostic effects of the profiling and to find the appropriate cutoff point, and to validate the procedure and cutoffs in the validation set.

\section{Statistical analysis}

Data are presented as mean (S.E.M.). Student's $t$-test and Mann-Whitney unpaired analysis of variance were used to evaluate statistical differences between patients and controls. Analysis of area under the ROC curve (AUC) was used to estimate the effectiveness of lncRNAs for predicting HC. Statistical analyses were performed using STATA 9.2 and GraphPad Prism software. In all cases, $\mathrm{P}<0.05$ was considered significant. All $P$ values were two-sided.

\section{Results}

Candidate IncRNA expression in HC plasma samples

Candidate lncRNAs were screened using the PubMed database through searching the terms "cholangiocarcinoma" and "IncRNA." This obtained 12 candidate IncRNAs: CCAT2, Sox2ot, UCA1, TUG1, AFAP1-AS1, CCAT1, NEAT1, PCAT1, PANDAR, MALAT1, H19, and CPS1IT1. We first detected these 12 candidates in plasma samples from $20 \mathrm{HC}$ patients and healthy controls (training set). Three of the 12 lncRNAs were detectable and significantly different in the plasma samples from HC patients and healthy controls. The remaining lncRNAs were filtered out. For example, UCA1, AFAP1-AS1, and H19 were in too low abundance for further investigation, while CCAT2, Sox2ot, TUG1, CCAT1, NEAT1, PCAT1, and PANDAR were not significantly different between patients and controls (Fig. 1). Thus, three lncRNAs (PCAT1, MALAT1 and CPS1-IT1) were further investigated in the validation set.

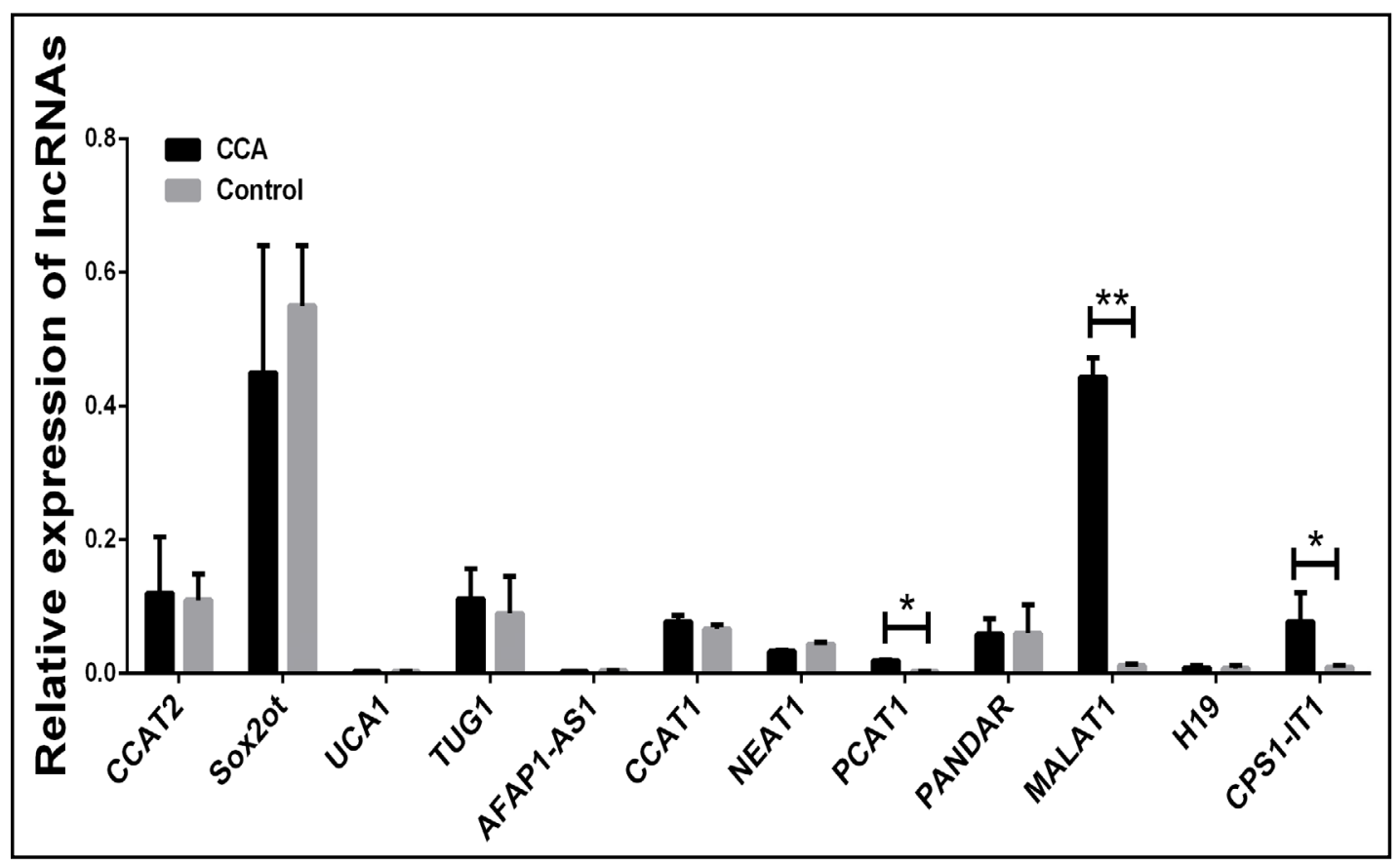

Fig. 1. Relative expression of the candidate IncRNAs. Total 20 paired plasma from HC patients and 20 controls were used in RT-qPCR analysis. Data was presented as mean \pm SEM. Data was analyzed with Student's t-test. *indicated $\mathrm{p}<0.05$ and ${ }^{* *}$ indicated $\mathrm{p}<0.01$.

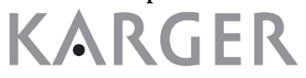




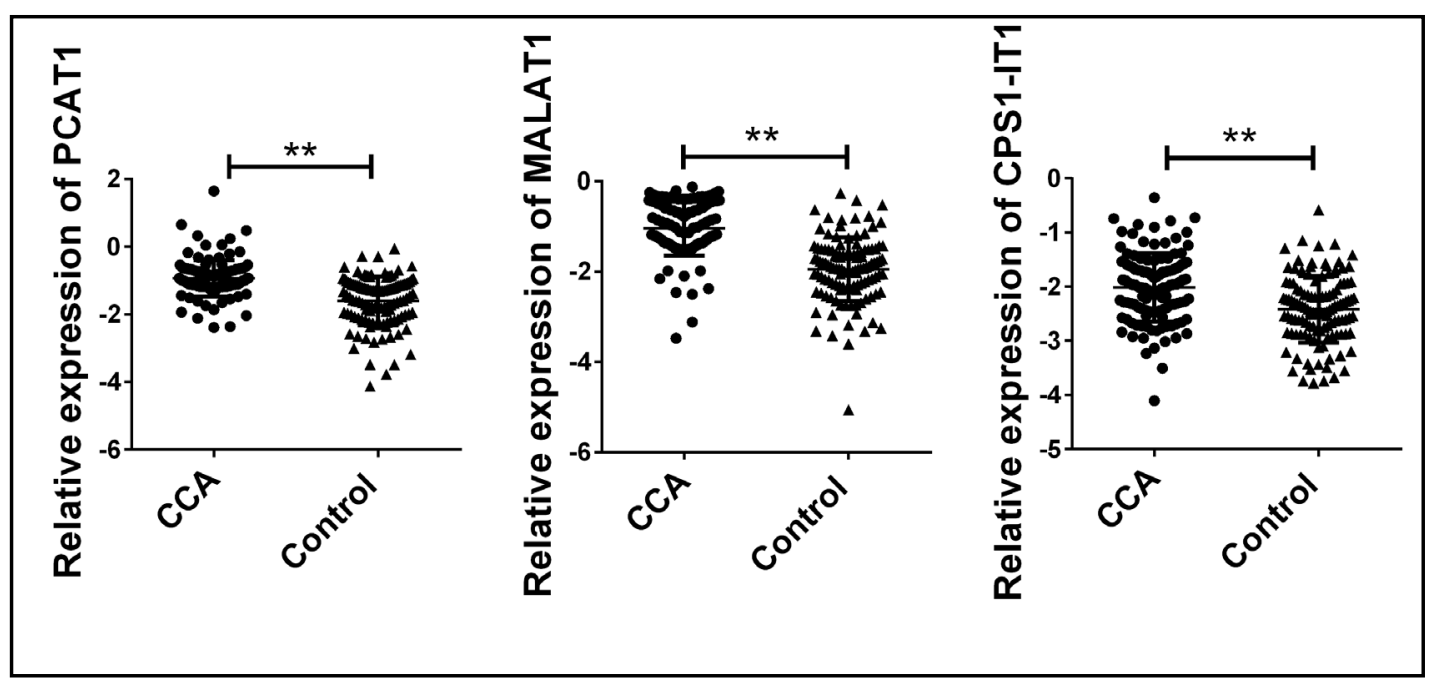

Fig. 2. Validation of lncRNAs expression in a larger sample size. Plasma from $120 \mathrm{HC}$ patients and 120 controls were enrolled. Data were presented as plot of the median and range of log-transformed relative expression level and was analyzed with Student's t-test. ${ }^{* *}$ indicated $\mathrm{p}<0.01$.

Increased PCAT1, MALAT1 and CPS1-IT1 levels in the validation set

We next examined the expression of these three IncRNAs in the larger validation cohort of 120 cases and controls. As shown in Fig. 2, we found that PCAT1, MALAT1, and CPS1-IT1 levels were consistent with the results of the training set: increased expression and stable abundance.

Risk score analysis for a potential HC fingerprint

A risk score formula was
Table 3. Risk score analysis of in HC and cancer-free control plasma samples. ${ }^{a} \mathrm{PPV}$, positive predictive value. ${ }^{\mathrm{b}} \mathrm{NPV}$, negative predictive value

\begin{tabular}{lcccc}
\hline Score & $0-7.883$ & $7.8833-11.525$ & $\mathrm{PPV}^{\mathrm{a}}$ & $\mathrm{NPVb}$ \\
\hline Training set & & & 0.800 & 0.800 \\
HC & 4 & 16 & & \\
Control & 16 & 4 & & \\
Validation set & & & 0.808 & 0.783 \\
HC & 23 & 97 & & \\
Control & 94 & 26 & & \\
\hline
\end{tabular}
applied to evaluate the diagnostic value of the three lncRNAs. First, we divided the control group and case group into a training set according to the upper $95 \%$ credibility interval $(95 \% \mathrm{CI})$ in the control group. The risk score was calculated based on the logistic regression analysis. All plasma samples were then divided into a high risk group which indicated the high possibility of being in the HC group, and a low risk group, representing predicted controls. We defined the cut-off value as the maximal value of sensitivity + specificity. The positive predictive value (PPV) and negative predictive value (NPV) were both calculated as $80.0 \%$ in the training set. We further applied the same value to calculate the risk score of samples in the validation set, for this analysis the PPV and NPV were $80.3 \%$ and $78.3 \%$, respectively (Table 3 ).

ROC curve analysis was used to evaluate predictive diagnostic values of IncRNAs for HC. The AUC of the training set of three IncRNAs (PCAT1, MALAT1 and CPS1-IT1) signature were $0.802,0.795$, and 0.660 , respectively, while the combination of the three factors possessed a moderate ability to discriminate between HCC patients and controls with an AUCe of 0.810 (Fig. 3A). The AUC of the validation set three lncRNAs signatures were $0.784,0.860$, and 0.677 , respectively, and the combination of the three factors possessed a moderate ability to discriminate between HC patients and controls with an AUC of 0.893 (Fig. 3B). 


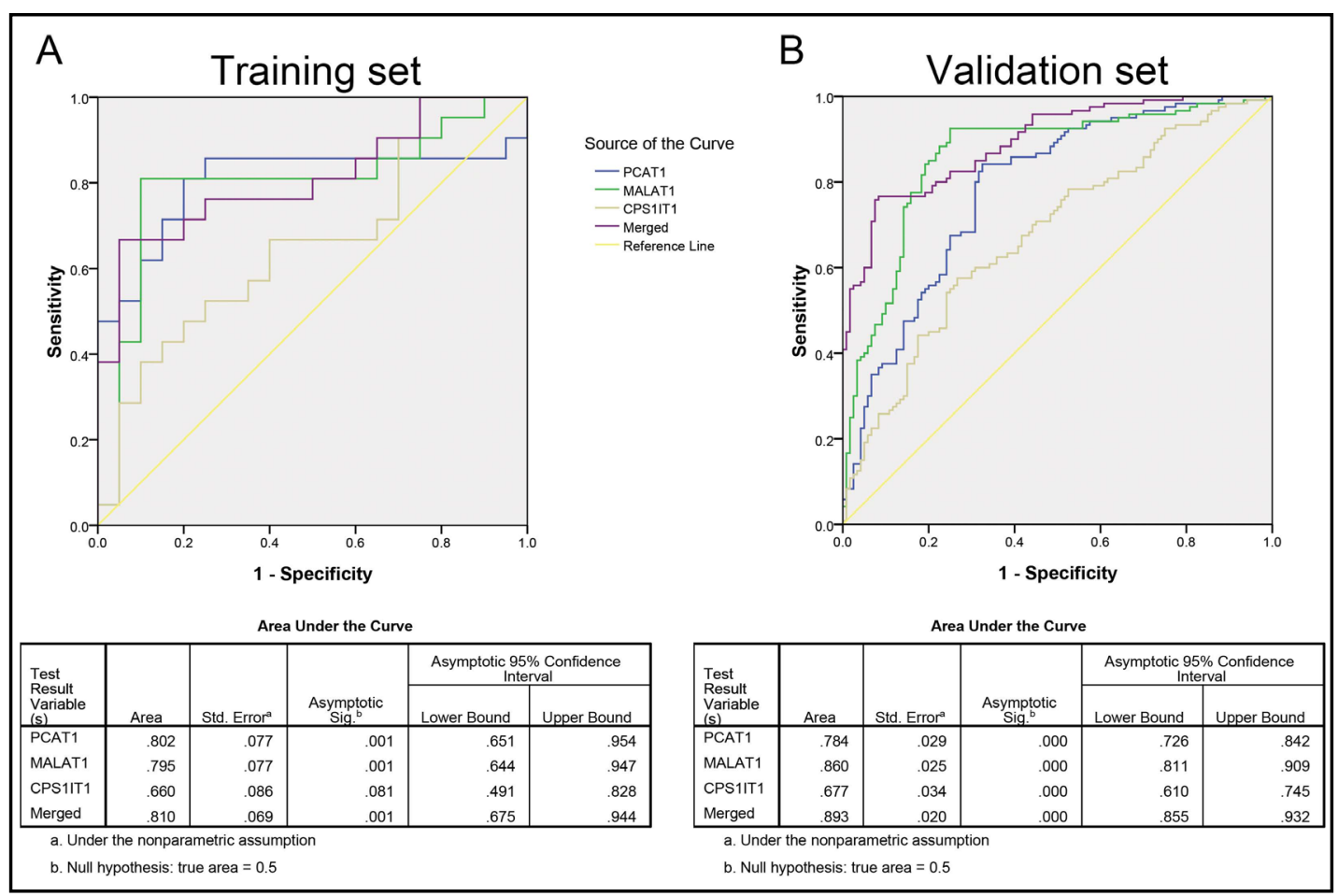

Fig. 3. ROC analysis of the three potential biomarkers for HC by using risk score analysis. A: ROC analysis of the three potential biomarkers for HC in training set, merged indicated the combination of three lncRNAs. $\mathrm{B}$ : ROC analysis of the three potential biomarkers for $\mathrm{HC}$ in validation set, merged indicated the combination of three lncRNAs.

Fig. 4. Stability detection of IncRNAs in human plasma samples. The comparative $\quad 2-\Delta \Delta \mathrm{Ct}$ method algorithm was used for data presentation, and the Student's t-test was used to analyze the difference. Sox2ot was used as the positive control based on the abundant endogenous expression. Data was presented as mean \pm SEM.

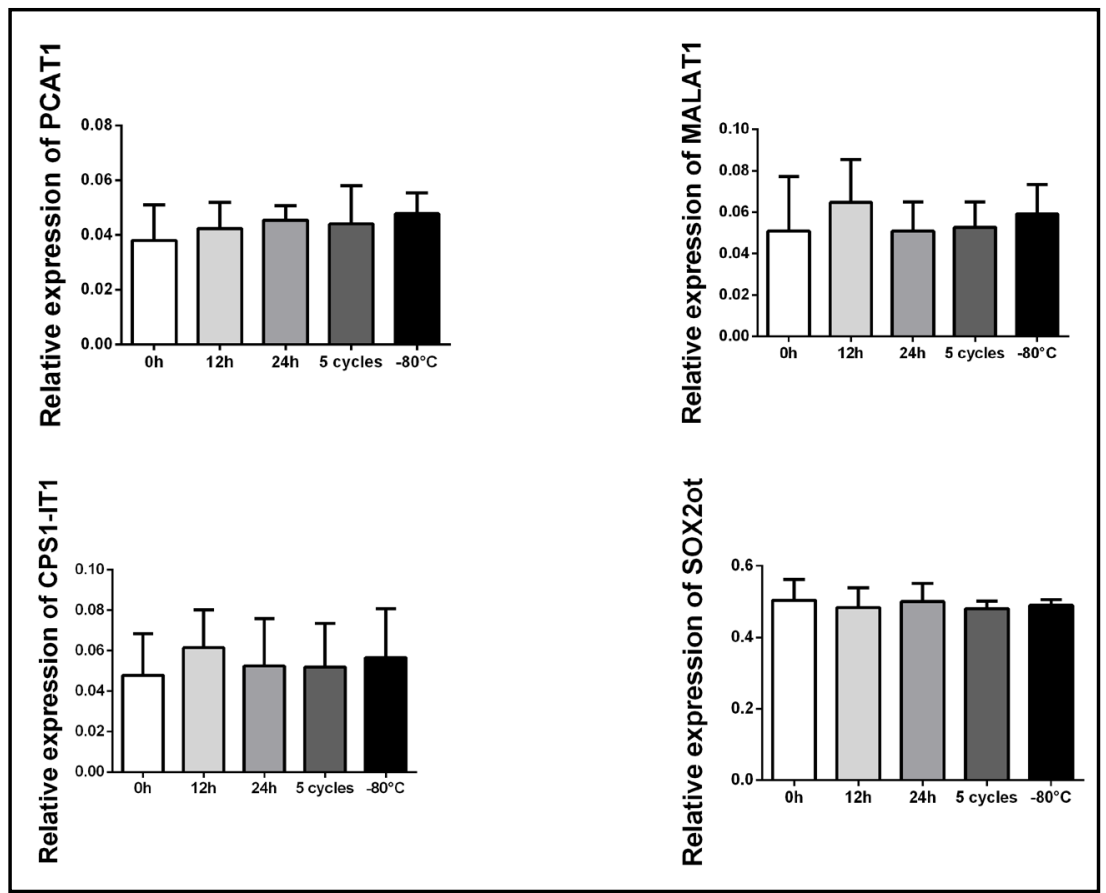




\section{Stability detection of IncRNAs in human plasma samples}

We next amplified the three IncRNAs in five healthy controls. We incubated human plasma obtained from three healthy controls at room temperature for 0,12 , and $24 \mathrm{~h}$ and subjected it to five freeze/thaw cycles before detecting the product by qRT-PCR. The relative expression of PCAT1, MALAT1, and CPS1-IT1 normalized to GAPDH are presented in Fig. 4 , indicating that all the three lncRNAs were detectable in human plasma. Sox2ot was used as the positive control based on its abundant endogenous expression. We found that the expression level of the three IncRNAs were altered, indicating that PCAT1, MALAT1, and CPS1-IT1 were stably expressed and detectable in human plasma.

\section{Discussion}

Despite the number of diagnostic advances and therapeutic strategies that have been achieved for HC in recent years, the overall survival rates of HC patients is very poor due to recurrence and metastasis [24]. Due to the lack of effective preventive measures and obvious symptoms, HC patients are usually diagnosed at advanced clinical stages with local and/or distant metastases [25]. Therefore, it is of great importance to identify efficient biomarkers that offer diagnostic, prognostic, and therapeutic strategies. The etiology and mutational pattern between intra- and extra-hepatic cholangiocarcinoma types are different. In this study, we only focused on HC.

The exploration of HC biomarkers has been undertaken by numerous researchers for decades. These studies have been revealed significantly elevated miR-21 in patients with intrahepatic HC, showing high discrimination ability between patients and healthy controls. Interestingly, serum miR-21 levels have been found to decrease after surgical resection. Furthermore, serum levels of miR-483-5p and miR-222 were able to discriminate between PSC and HC patients [26]. Not only miRNAs, but also lncRNAs have been identified as potential biomarkers for predicting the occurrence or development of human malignant tumors. For example, circulating serum Linc00974 has been proven to be a biomarker for the early screening of hepatocellular carcinoma [27]; however, such clinical studies on circulating lncRNAs in HC have not been adequately performed.

In this study, there were a total of 12 IncRNAs that were included as biomarker candidates based on their previously reported functions. These specific lncRNAs were differentially expressed in HC tissues, and therefore might present altered levels in patients' serum samples. Previous studies have speculated that aberrantly expressed circulating lncRNAs might be derived from tumors. Thus, we examined the expression of the 12 lncRNAs in patients and healthy controls. By employing a multiple screening phase including training and validation sets, we found that only PCAT1, MALAT1, and CPS1-IT1 were differentially expressed in HC (increased). Upregulated PCAT1 in HC tumor tissues acts as a competing endogenous for miR-122, increasing levels of glycogen synthase kinase $3 \beta$ and decreasing $\beta$-catenin levels in whole cell lysates and nuclear fractions, indicating that PCAT1 silencing inhibited Wnt/ $\beta$-catenin-signaling, promoting cell invasion $[28,29]$. LncRNA MALAT1 also promotes HC cell proliferation and invasion by activating the PI3K/Akt pathway [29]. For CPS1-IT1, CPS1, and CPS1-IT1 overexpression was correlated with increased CA19-9 positivity and lymph node metastasis [30]. Additionally, upregulation of CPS1 and CPS1-IT1 has a negative impact on the overall survival rate of HC patients. By employing the risk score analysis, we found that the combination of the three factors presented a high sensitivity and specificity with an AUC of 0.893 , indicating that PCAT1, MALAT1, and CPS1-IT1 might serve as a signature for predicting $\mathrm{HC}$. 


\section{Cellular Physiology Cell Physiol Biochem 2018;49:1694-1702

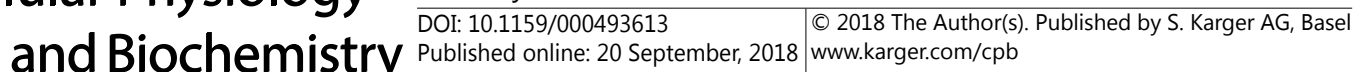

Shi et al.: Biomarker in $\mathrm{HC}$

\section{Conclusion}

In summary, we identified three IncRNAs, PCAT1, MALAT1, and CPS1-IT1, as the potential biomarkers for the tumorigenesis prediction of $\mathrm{HC}$ in this study. Due to the sample size limitation, our study were a preliminary work, a deeper investigation regarding the potential mechanism of the three lncRNAs in the occurrence or development of $\mathrm{HC}$ remains to be explored in the future.

\section{Disclosure Statement}

The authors declare that no conflict of interest exist.

\section{References}

1 Nepal C, O’Rourke CJ, Oliveira DV, Taranta A, Shema S, Gautam P, Calderaro J, Barbour A, Raggi C, Wennerberg K, Wang XW, Lautem A, Roberts LR, Andersen JB: Genomic Perturbations Reveal Distinct Regulatory Networks in Intrahepatic Cholangiocarcinoma. Hepatology 2017;10.1002/hep.29764.

2 Lei Z, Xia Y, Si A, Wang K, Li J, Yan Z, Yang T, Wu D, Wan X, Zhou W, Liu J, Wang H, Cong W, Wu M, Pawlik TM, Lau WY, Shen F: Antiviral Therapy Improves Survival in patients with HBV infection and Intrahepatic Cholangiocarcinoma undergoing Liver Resection. J Hepatol 2017;10.1016/j.jhep.2017.11.015.

- 3 Dong M, Liu X, Evert K, Utpatel K, Peters M, Zhang S, Xu Z, Che L, Cigliano A, Ribback S, Dombrowski F, Cossu A, Gordan J, Calvisi DF, Evert M, Liu Y, Chen X: Efficacy of MEK inhibition in a K-Ras-driven cholangiocarcinoma preclinical model. Cell Death Dis 2018;9:31.

-4 Jones MR, Lim H, Shen Y, Pleasance E, Ch'ng C, Reisle C, Leelakumari S, Zhao C, Yip S, Ho J, Zhong E, Ng T, Ionescu D, Schaeffer DF, Mungall AJ, Mungall KL, Zhao Y, Moore RA, Ma Y, Chia S et al.: Successful targeting of the NRG1 pathway indicates novel treatment strategy for metastatic cancer. Ann Oncol 2017;28:30923097.

5 Le Roy B, Gelli M, Pittau G, Allard MA, Pereira B, Serji B, Vibert E, Castaing D, Adam R, Cherqui D, Sa Cunha A: Neoadjuvant chemotherapy for initially unresectable intrahepatic cholangiocarcinoma. Br J Surg 2017;10.1002/bjs.10641.

6 Yin Y, Zhang M, Dorfman RG, Li Y, Zhao Z, Pan Y, Zhou Q Huang S, Zhao S, Yao Y, Zou X: Histone deacetylase 3 overexpression in human cholangiocarcinoma and promotion of cell growth via apoptosis inhibition. Cell Death Dis 2017;8:e2856.

7 Bagante F, Merath K, Squires MH, Weiss M, Alexandrescu S, Marques HP, Aldrighetti L, Maithel SK, Pulitano C, Bauer TW, Shen F, Poultsides GA, Soubrane O, Martel G, Groot Koerkamp B, Guglielmi A, Itaru E, Pawlik TM: The Limitations of Standard Clinicopathologic Features to Accurately Risk-Stratify Prognosis after Resection of Intrahepatic Cholangiocarcinoma. J Gastrointest Surg 2018;22:477-485.

$>8$ Peraldo-Neia C, Cavalloni G, Fenocchio E, Cagnazzo C, Gammaitoni L, Cereda S, Nasti G, Satolli MA, Aprile G, Reni M, Avallone A, Spadi R, Venesio T, Martin V, Doglioni C, Frattini M, Aglietta M, Leone F: Prognostic and predictive role of EGFR pathway alterations in biliary cancer patients treated with chemotherapy and antiEGFR. PLoS One 2018;13:e0191593.

-9 Kim BH, Kim E, Kim K, Jang JY, Kim SW, Oh DY, Chie EK: The impact of perioperative CA19-9 change on the survival and recurrence patterns after adjuvant chemoradiotherapy in resectable extrahepatic cholangiocarcinoma. J Surg Oncol 2018;117:380-388.

10 Zhou Y, Zhou Q Lin Q, Chen R, Gong Y, Liu Y, Yu M, Zeng B, Li K, Chen R, Li Z: Evaluation of risk factors for extrahepatic cholangiocarcinoma: ABO blood group, hepatitis B virus and their synergism. Int J Cancer 2013;133:1867-1875.

-11 Askarian-Amiri ME, Seyfoddin V, Smart CE, Wang J, Kim JE, Hansji H, Baguley BC, Finlay GJ, Leung EY: Emerging role of long non-coding RNA SOX2OT in SOX2 regulation in breast cancer. PLoS One 2014;9:e102140. 


\section{Cellular Physiology Cell Physiol Biochem 2018;49:1694-1702 and Biochemistry \begin{tabular}{c|c|c|} 
DOI: 10.1159/000493613 & O 2018 The Author(s). Published by S. Karger AG, Basel \\
wubw.karger.com/cpb
\end{tabular}

12 Gong J, Tian J, Lou J, Ke J, Li L, Li J, Yang Y, Gong Y, Zhu Y, Zhang Y, Zhong R, Chang J, Miao X: A functional polymorphism in lnc-LAMC2-1:1 confers risk of colorectal cancer by affecting miRNA binding. Carcinogenesis 2016;10.1093/carcin/bgw024.

13 Li J, Wang X, Tang J, Jiang R, Zhang W, Ji J, Sun B: HULC and Linc00152 Act as Novel Biomarkers in Predicting Diagnosis of Hepatocellular Carcinoma. Cell Physiol Biochem 2015;37:687-696.

14 Tong YS, Wang XW, Zhou XL, Liu ZH, Yang TX, Shi WH, Xie HW, Lv J, Wu QQ, Cao XF: Identification of the long non-coding RNA POU3F3 in plasma as a novel biomarker for diagnosis of esophageal squamous cell carcinoma. Mol Cancer 2015;14:3.

15 Zhou X, Ye F, Yin C, Zhuang Y, Yue G, Zhang G: The Interaction Between MiR-141 and lncRNA-H19 in Regulating Cell Proliferation and Migration in Gastric Cancer. Cell Physiol Biochem 2015;36:1440-1452.

16 Ji J, Tang J, Deng L, Xie Y, Jiang R, Li G, Sun B: LINC00152 promotes proliferation in hepatocellular carcinoma by targeting EpCAM via the mTOR signaling pathway. Oncotarget 2015;6:42813-42824.

17 Wang Y, Wu P, Lin R, Rong L, Xue Y, Fang Y: LncRNA NALT interaction with NOTCH1 promoted cell proliferation in pediatric T cell acute lymphoblastic leukemia. Sci Rep 2015;5:13749.

18 Jiang R, Tang J, Chen Y, Deng L, Ji J, Xie Y, Wang K, Jia W, Chu WM, Sun B: The long noncoding RNA lnc-EGFR stimulates T-regulatory cells differentiation thus promoting hepatocellular carcinoma immune evasion. Nat Commun 2017;8:15129.

19 Yang JP, Yang XJ, Xiao L, Wang Y: Long noncoding RNA PVT1 as a novel serum biomarker for detection of cervical cancer. Eur Rev Med Pharmacol Sci 2016;20:3980-3986.

20 Lorenzen JM, Schauerte C, Kolling M, Hubner A, Knapp M, Haller H, Thum T: Long Noncoding RNAs in Urine Are Detectable and May Enable Early Detection of Acute T Cell-Mediated Rejection of Renal Allografts. Clin Chem 2015;61:1505-1514.

-21 Yan Y, Zhang B, Liu N, Qi C, Xiao Y, Tian X, Li T, Liu B: Circulating Long Noncoding RNA UCA1 as a Novel Biomarker of Acute Myocardial Infarction. Biomed Res Int 2016;2016:8079372.

22 Tang J, Jiang R, Deng L, Zhang X, Wang K, Sun B: Circulation long non-coding RNAs act as biomarkers for predicting tumorigenesis and metastasis in hepatocellular carcinoma. Oncotarget 2015;6:4505-4515.

23 Ebrahimi S, Hosseini M, Ghasemi F, Shahidsales S, Maftouh M, Akbarzade H, Parizadeh SA, Hassanian SM, Avan A: Circulating microRNAs as Potential Diagnostic, Prognostic and Therapeutic Targets in Pancreatic Cancer. Curr Pharm Des 2016;22:6444-6450.

24 Xie N, Cai JB, Zhang L, Zhang PF, Shen YH, Yang X, Lu JC, Gao DM, Kang Q Liu LX, Zhang C, Huang XY, Zou H, Zhang XY, Song ZJ, Sun HX, Fu BM, Ke AW, Shi GM: Upregulation of B7-H4 promotes tumor progression of intrahepatic cholangiocarcinoma. Cell Death Dis 2017;8:3205.

25 Lee ES, Jeong MS, Singh R, Jung J, Yoon H, Min JK, Kim KH, Hong HJ: A chimeric antibody to L1 cell adhesion molecule shows therapeutic effect in an intrahepatic cholangiocarcinoma model. Exp Mol Med 2012;44:293-302.

26 Caviglia JM, Yan J, Jang MK, Gwak GY, Affo S, Yu L, Olinga P, Friedman RA, Chen X, Schwabe RF: MicroRNA-21 and Dicer are dispensable for hepatic stellate cell activation and the development of liver fibrosis. Hepatology 2017;10.1002/hep.29627.

27 Tang J, Zhuo H, Zhang X, Jiang R, Ji J, Deng L, Qian X, Zhang F, Sun B: A novel biomarker Linc00974 interacting with KRT19 promotes proliferation and metastasis in hepatocellular carcinoma. Cell Death Dis 2014;5:e1549.

-28 Zhang F, Wan M, Xu Y, Li Z, Leng K, Kang P, Cui Y, Jiang X: Long noncoding RNA PCAT1 regulates extrahepatic cholangiocarcinoma progression via the Wnt/beta-catenin-signaling pathway. Biomed Pharmacother 2017;94:55-62.

29 Wang C, Mao ZP, Wang L, Wu GH, Zhang FH, Wang DY, Shi JL: Long non-coding RNA MALAT1 promotes cholangiocarcinoma cell proliferation and invasion by activating PI3K/Akt pathway. Neoplasma 2017;64:725-731.

-30 Wang TH, Wu CH, Yeh CT, Su SC, Hsia SM, Liang KH, Chen CC, Hsueh C, Chen CY: Melatonin suppresses hepatocellular carcinoma progression via IncRNA-CPS1-IT-mediated HIF-1alpha inactivation. Oncotarget 2017;8:82280-82293. 